\title{
Fully-differential NNLO predictions for vector-boson pair production with MATRIX
}

\author{
Massimiliano Grazzini, Marius Wiesemann* \\ Physics Department, University of Zürich, \\ Winterthurerstrasse 190, CH-8057 Zürich, Switzerland \\ E-mail: grazzini@physik.uzh.ch, mariusw@physik.uzh.ch
}

\section{Stefan Kallweit}

PRISMA Cluster of Excellence, Institute of Physics, Johannes Gutenberg University,

D-55099 Mainz, Germany

E-mail: kallweiteuni-mainz.de

\section{Dirk Rathlev}

Theory Group, Deutsches Elektronen-Synchrotron (DESY), D-22607 Hamburg, Germany

E-mail: dirk.rathlevedesy.de

\begin{abstract}
We review the computations of the next-to-next-to-leading order (NNLO) QCD corrections to vector-boson pair production processes in proton-proton collisions and their implementation in the numerical code MATRIX. Our calculations include the leptonic decays of $W$ and $Z$ bosons, consistently taking into account all spin correlations, off-shell effects and non-resonant contributions. For massive vector-boson pairs we show inclusive cross sections, applying the respective mass windows chosen by ATLAS and CMS to define $Z$ bosons from their leptonic decay products, as well as total cross sections for stable bosons. Moreover, we provide samples of differential distributions in fiducial phase-space regions inspired by typical selection cuts used by the LHC experiments. For the vast majority of measurements, the inclusion of NNLO corrections significantly improves the agreement of the Standard Model predictions with data.
\end{abstract}

Loops and Legs in Quantum Field Theory

24-29 April 2016

Leipzig, Germany

\footnotetext{
* Speaker.
} 


\section{Introduction}

Vector-boson pair production at the Large Hadron Collider (LHC) provides an important test of the electroweak (EW) sector of the Standard Model (SM) at the TeV scale. As these processes are sensitive to the gauge-boson self-interactions, any small deviation in the observed rates or in the kinematical distributions could give a hint towards new physics, which can be modelled through anomalous couplings. Vector-boson pair production processes also constitute backgrounds in many direct new-physics searches, and, in Higgs-boson studies, the neutral final states represent irreducible backgrounds in the respective decay channels $H \rightarrow Z Z / W^{+} W^{-} / Z \gamma$.

In this contribution, we give an overview of the applications of the MATRIX code to vectorboson pair production processes at NNLO QCD accuracy. Besides a summary of the most relevant phenomenological results for the inclusive cross sections and distributions, we discuss the implementation of automated $q_{T}$ subtraction (and resummation) for both the quark- and gluon-induced production of colour singlets and review the status as well as the capabilities of the code.

\section{NNLO corrections through automated $\mathbf{q}_{\mathrm{T}}$ subtraction}

The cancellation of singularities across the various IR-divergent amplitudes, different with respect to their parton multiplicities, in an NNLO computation is a highly non-trivial task. Suitable subtraction schemes have been devised to deal with the additional complications arising at NNLO with respect to next-to-leading order (NLO). In order to achieve automation in a numerical code, such schemes must fulfill certain requirements: Firstly, they should cover a reasonable number of applicable processes; their formulation must be unspecific to the process; the procedure must be sufficiently simple to render a numerical implementation feasible; their numerical convergence may not exceed the limits set by the current technological status of computers/clusters. All these features perfectly apply to the $q_{T}$ subtraction formalism.

At variance with the fully local subtraction methods, such as antenna subtraction [1, 2, 3, 4], colourful subtraction $[5,6,7]$ and STRIPPER $[8,9,10], q_{T}$ subtraction corresponds to a non-local subtraction scheme. It starts from an NLO calculation with one additional parton (jet) in the final state, making use of the highly sophisticated automation and numerical control we have by now on the computation of NLO cross sections, and devises suitable subtractions of the remaining logarithmic divergences in $q_{T}$ to render the cross section finite at NNLO QCD accuracy.

The $q_{T}$-subtraction formalism [11] deals with the production of an arbitrary number of colour singlets $^{1}$ at hadron colliders. Denoting the system of colourless final states by $F$, the $p p \rightarrow F+X$ cross section at $(\mathrm{N}) \mathrm{NLO}$ in this framework can be written as

$$
\mathrm{d} \sigma_{(\mathrm{N}) \mathrm{NLO}}^{F}=\left[\mathrm{d} \sigma_{(\mathrm{N}) \mathrm{LO}}^{F+\mathrm{jet}}-\mathrm{d} \sigma_{(\mathrm{N}) \mathrm{NLO}}^{\mathrm{CT}}\right]+\mathscr{H}_{(\mathrm{N}) \mathrm{NLO}}^{F} \otimes \mathrm{d} \sigma_{\mathrm{LO}}^{F}
$$

The term $d \sigma_{(\mathrm{N}) \mathrm{LO}}^{F+\text { jet }}$ represents the cross section for the production of the system $F$ plus one jet at $(\mathrm{N}) \mathrm{LO}$ accuracy. The counterterm $\mathrm{d} \sigma_{(\mathrm{N}) \mathrm{NLO}}^{\mathrm{CT}}$ is obtained from the fixed-order expansion of the resummation formula for logarithmically enhanced contributions at small transverse momenta [15]

\footnotetext{
${ }^{1}$ The extension to heavy-quark production has been discussed in Ref. [12]. Furthermore, the basic idea of $q_{T}$ subtraction has recently been extended to processes including light partons at the leading order by using the $N$-jettiness observable instead of the transverse momentum of the colourless system [13, 14].
} 
and guarantees the cancellation of the remaining IR divergences of the $F+$ jet cross section. The hard-collinear coefficient $\mathscr{H}_{(\mathrm{N}) \mathrm{NLO}}^{F}$ is a perturbative function in $\alpha_{\mathrm{S}}$, whose general structure is known at NLO [16] and NNLO [17]. ${ }^{2} \mathscr{H}_{(\mathrm{N}) \mathrm{NLO}}^{F}$ entails the one-loop (and two-loop) virtual corrections to the Born-level process and compensates for the subtraction of $\mathrm{d} \sigma_{(\mathrm{N}) \mathrm{NLO}}^{\mathrm{CT}}$. The last term of Eq. (2.1) therefore adds all the contributions missing at vanishing transverse momentum of $F$ to obtain full (N)NLO QCD accuracy.

In the same framework automated transverse-momentum resummation of the colourless system is obtained by replacing the last term of Eq. (2.1) by the suitable small- $p_{\mathrm{T}}$ resummation formula of Ref. [15]. The latter is independent of the respective process apart from the inclusion of the $\mathscr{H}_{(\mathrm{N}) \mathrm{NLO}}^{F}$ coefficient, whose process dependence is fully determined by the loop corrections to the Born-level process.

\section{The MATRIX code}

MATRIX $^{3}$ is a numerical code based on the Monte Carlo program MUNICH ${ }^{4}$, interfaced with the OPENLOOPS ${ }^{5}$ generator of one-loop scattering amplitudes [20], and includes an automated implementation of $q_{T}$-subtraction and -resummation, as introduced in the previous section. This widely automated framework is limited only by the two-loop amplitudes entering $\mathscr{H}_{\mathrm{NNLO}}^{F}$ in Eq. (2.1), and has already been used, in combination with the two-loop scattering amplitudes of Refs. [21, 22], for the calculations of $Z \gamma$ [23, 24], $W^{ \pm} \gamma$ [24], $Z Z$ [25, 26], $W^{+} W^{-}$[27, 28] and $W^{ \pm} Z$ [29] production at NNLO QCD as well as in the resummed computations of the $Z Z$ and $W^{+} W^{-}$transverse-momentum spectra [30] at NNLL+NNLO.

The MATRIX code is currently in a closed beta phase and is already used by several experimental groups for direct physics applications. A public release of the code is currently in preparation and should be available in near future. The code features a large number of single and double vector-boson and Higgs-production processes listed in Tab. 1. The code is engineered in a very user-friendly way that guides you from the very first execution of MATRIX to the very end of a run of a specific process.

Below, we summarize the basic steps to compile and run the code. Further details will be available in the manual shipped with the code.

The command

$\$ . /$ matrix

will result in entering the MATRIX shell, an interactive steering interface for the compilation and the setup of a certain process. Next we can choose one ID of the many processes from the list in Tab. 1, e.g.,

\footnotetext{
${ }^{2}$ The latter exploits the explicit NNLO results for Higgs [18] and vector-boson [19] production.

${ }^{3}$ MATRIX is the abbreviation of "MUNICH Automates qT subtraction and Resummation to Integrate X-sections", by M. Grazzini, S. Kallweit, D. Rathlev, and M. Wiesemann. In preparation.

${ }^{4}$ MUNICH is the abbreviation of "MUlti-chaNnel Integrator at Swiss $(\mathrm{CH})$ precision" - an automated parton level NLO generator by S. Kallweit. In preparation.

${ }^{5}$ The OPENLOOPS one-loop generator, by F. Cascioli, J. Lindert, P. Maierhöfer, and S. Pozzorini, is publicly available at http: //openloops.hepforge.org.
} 


\begin{tabular}{lll}
\hline ID & process & description \\
\hline pph21 & $p p \rightarrow H$ & on-shell Higgs boson production \\
ppz01 & $p p \rightarrow Z$ & on-shell $Z$ production \\
ppw01 & $p p \rightarrow W^{-}$ & on-shell $W^{-}$production \\
ppwx01 & $p p \rightarrow W^{+}$ & on-shell $W^{+}$production \\
ppeex02 & $p p \rightarrow e^{-} e^{+}$ & $Z$ production with decay \\
ppnenex02 & $p p \rightarrow v_{e} \bar{v}_{e}$ & $Z$ production with decay \\
ppenex02 & $p p \rightarrow e^{-} \bar{v}_{e}$ & $W^{-}$production with decay \\
ppexne02 & $p p \rightarrow e^{+} v_{e}$ & $W^{+}$production with decay \\
pphh22 & $p p \rightarrow H H$ & on-shell double Higgs boson production \\
ppaa02 & $p p \rightarrow \gamma \gamma$ & on-shell $\gamma \gamma$ production \\
ppzz02 & $p p \rightarrow Z Z$ & on-shell $Z Z$ production \\
ppwxw02 & $p p \rightarrow W^{+} W^{-}$ & on-shell $W^{+} W^{-}$production \\
ppeexa03 & $p p \rightarrow e^{-} e^{+} \gamma$ & $Z \gamma$ production with decay \\
ppnenexa03 & $p p \rightarrow v_{e} \bar{v}_{e} \gamma$ & $Z \gamma$ production with decay \\
ppenexa03 & $p p \rightarrow e^{-} \bar{v}_{e} \gamma$ & $W^{-} \gamma$ with decay \\
ppexnea03 & $p p \rightarrow e^{+} v_{e} \gamma$ & $W^{+} \gamma$ with decay \\
ppemexmx04 & $p p \rightarrow e^{-} \mu^{-} e^{+} \mu^{+}$ & $Z Z$ production with decay \\
ppeeexex04 & $p p \rightarrow e^{-} e^{-} e^{+} e^{+}$ & $Z Z$ production with decay \\
ppeexnmnmx04 & $p p \rightarrow e^{-} e^{+} v_{\mu} \bar{v}_{\mu}$ & $Z Z$ production with decay \\
ppemxnmnex04 & $p p \rightarrow e^{-} \mu^{+} v_{\mu} \bar{v}_{e}$ & $W^{+} W^{-}$production with decay \\
ppeexnenex04 & $p p \rightarrow e^{-} e^{+} v_{e} \bar{v}_{e}$ & $Z Z / W^{+} W^{-}$production with decay \\
ppemexnmx04 & $p p \rightarrow e^{-} \mu^{-} e^{+} \bar{v}_{\mu}$ & $W^{-} Z$ production with decay \\
ppeeexnex04 & $p p \rightarrow e^{-} e^{-} e^{+} \bar{v}_{e}$ & $W^{-} Z$ production with decay \\
ppeexmxnm04 & $p p \rightarrow e^{-} e^{+} \mu^{+} v_{\mu}$ & $W^{+} Z$ production with decay \\
ppeexexne04 & $p p \rightarrow e^{-} e^{+} e^{+} v_{e}$ & $W^{+} Z$ production with decay \\
\hline
\end{tabular}

Table 1: Available processes in MATRIX.

|===> ppemxnmnex 04

for off-shell $W^{+} W^{-}$production in the different-flavor channel, which will automatically compile the code for this process including all relevant libraries, and generate the process folder for the runs. We now change the directory to the process folder, enter the MATRIX run shell, choose a name for the run (here "run_WW_process") and start running to create physics results by entering

\$ cd run/ppemxnmnex04_MATRIX

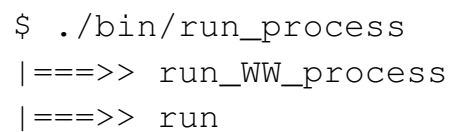

By default this will compute the LO cross section, no human intervention is needed from now on. Once the run is finished the results are collected in the dedicated folder. Additionally, the most relevant results (total rates) are printed on the screen.

To adjust certain inputs, the interactive run shell can be used to modify the relevant input files. For example, the computation of NLO and NNLO accurate cross section predictions can be enabled 
in the parameter card, which can be entered after naming the run (and before starting the run) by typing

$\mid==>>$ parameter

in the MATRIX run shell.

The MATRIX code allows for two different running modes: local and cluster. Local running should work on any UNIX based machine and exploits all cores available on the machine by means of parallelization. The cluster running mode, entails a dedicated list of clusters, which can be chosen by the user, including certain cluster-specific settings. The submission of jobs to the cluster is then taken care of by the code automatically. In the current version MATRIX supports three standard clusters: LSF (e.g, lxplus), slurm and condor. ${ }^{6}$ PBS cluster support is currently under validation. Further clusters can be added through an easy python module.

\section{Results}

All calculations reviewed here have been carried out in the MATRIX framework introduced in the previous section.

In Figure 1 we provide predictions for the inclusive $Z Z, W^{+} W^{-}$and $W^{ \pm} Z$ cross sections at different orders in QCD perturbation theory for the relevant centre-of-mass energies. All these results are calculated with NNPDF3.0 parton distribution functions (PDFs) [31], and compared to the cross sections determined by ATLAS and CMS from their respective measurements. Our findings are briefly discussed in Sections 4.2-4.4. The histograms in Figures 2-4 are taken from the original publications and thus use the respective input parameters as specified therein.

\subsection{Fiducial cross sections and differential distributions for $Z \gamma$ and $W^{ \pm} \gamma$ production}

Measurements of $V \gamma$ final states have been carried out by ATLAS and CMS using the data sets at centre-of-mass energies of $7 \mathrm{TeV}[32,33,34]$ and $8 \mathrm{TeV}[35,36]$. Due to the massless photon in the final state, a total cross section cannot be defined. Instead, we investigate cross sections in the fiducial phase-space regions chosen by the experiments (see Refs. [23, 24]). The higher-order corrections for $W^{ \pm} \gamma$ are significantly larger than those for $Z \gamma$ : This can be traced back to a suppression of the $W^{ \pm} \gamma$ Born contributions due to a radiation zero, which is broken only beyond leading order (LO). The loop-induced gluon-gluon contribution to $Z \gamma$ production amounts to only about $10 \%$ of the $\mathscr{O}\left(\alpha_{\mathrm{s}}^{2}\right)$ corrections. We find significantly larger corrections if the applied selection cuts suppress resonant configurations with the photon emitted from the final-state leptons in Born kinematics. As expected, a jet veto results in a serious reduction of the higher-order effects. The agreement with experimental data is significantly improved, in particular for inclusive $W^{ \pm} \gamma$ production. Figure 2 illustrates these findings by means of the transverse-momentum distribution of the photon in $Z \gamma$ and $W^{ \pm} \gamma$ production.

\subsection{Inclusive cross sections and normalized differential distributions for $Z Z$ production}

Various measurements of $Z Z$ hadroproduction have been carried out at the LHC by both ATLAS and CMS at centre-of-mass energies of $7 \mathrm{TeV}[38,39], 8 \mathrm{TeV}[40,37]$ and $13 \mathrm{TeV}[41,42]$,

\footnotetext{
${ }^{6}$ The setup of the code requires the execution of the runs from a disk shared among the frontend and the nodes.
} 

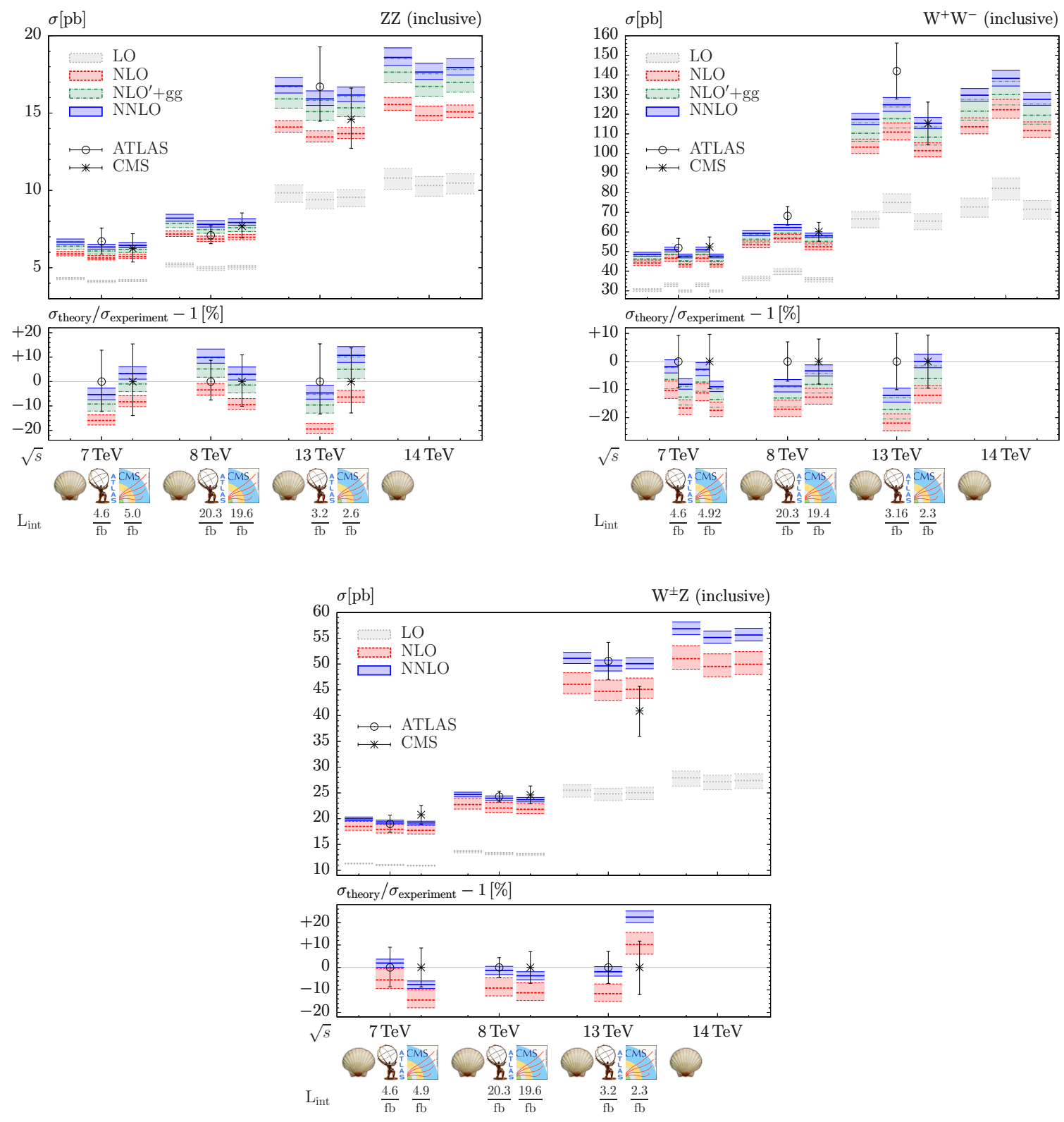

Figure 1: Total cross sections at LO, NLO, $\mathrm{NLO}^{\prime}+g g$ (NLO plus loop-induced $g g$ contribution, evaluated with NNLO PDFs) and NNLO for $Z Z$ (left), $W^{+} W^{-}$(center) and $W^{ \pm} Z$ (right) production, with uncertainties from conventional 7-point scale variations, are shown and compared to experimental results from ATLAS and CMS, where available. For each collider energy, the left column refers to cross sections evaluated with on-shell $W$ and/or $Z$ bosons, while the second (third) column gives fully inclusive off-shell results for fourlepton final states, corrected for their branching ratios, with only mass-window cuts corresponding to the respective ATLAS (CMS) analyses applied. In case of $W^{+} W^{-}$production, the $H \rightarrow W W^{*}$ production cross section predicted in NNLO QCD (from Ref. [28]) is added to the ATLAS predictions for $\sqrt{s}=8,13$ and $14 \mathrm{TeV}$, but not to the CMS predictions, in line with the published experimental setups. At $\sqrt{s}=7 \mathrm{TeV}$ results both with (left) and without (right) the Higgs-mediated contribution are shown. 

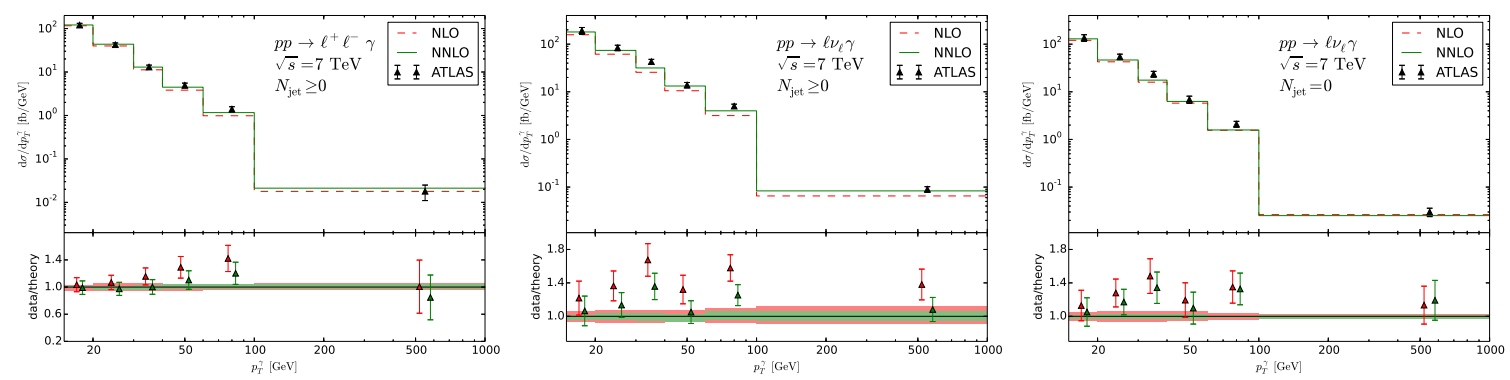

Figure 2: Distributions in the transverse momentum of the photon in $Z \gamma$ production (left), and in $W^{ \pm} \gamma$ production without (center) and with (right) a jet veto applied are shown, and compared to ATLAS data [32].
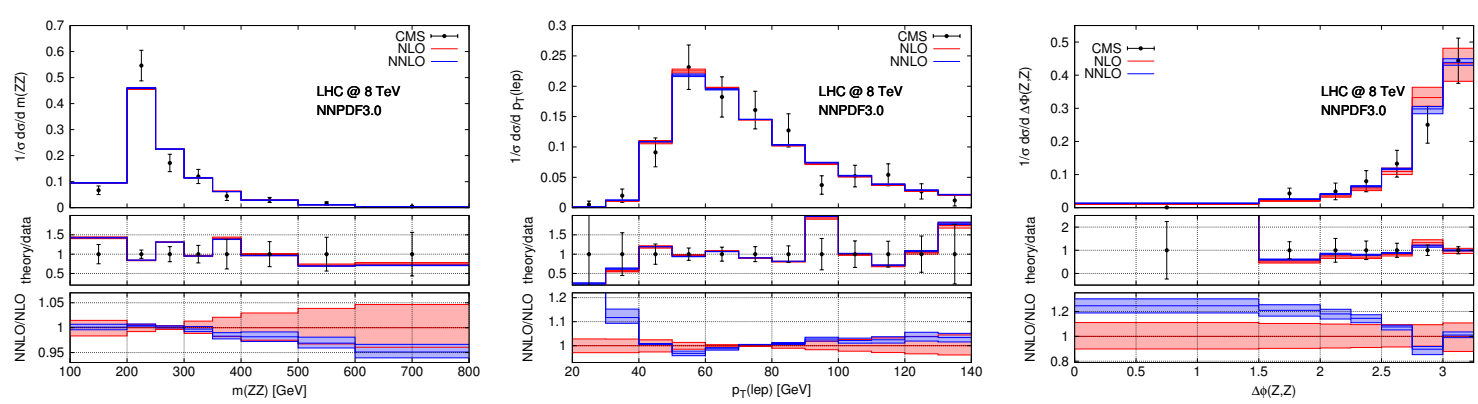

Figure 3: Normalized distributions in the four-lepton invariant mass (left), the leading-lepton $p_{\mathrm{T}}$ (center) and the azimuthal angle between the two reconstructed $Z$ bosons (right) are shown, and compared to CMS data [37].

which are in good agreement with NNLO QCD predictions. With typical definitions of fiducial phase-space regions, the higher-order corrections within fiducial cuts [26] mimick those ones found for the fully inclusive results [25] (see Figure 1). The loop-induced gluon-gluon contribution amounts to about $60 \%$ of the full $\mathscr{O}\left(\alpha_{\mathrm{s}}^{2}\right)$ corrections. In Figure 3 we show normalized distributions in the four-lepton invariant mass, the leading-lepton $p_{\mathrm{T}}$ and the azimuthal angle between the two reconstructed $Z$ bosons. Due to the large experimental uncertainties, a slightly improved shape agreement can be found only for the last one, which is non-trivial only beyond LO, and thus more affected by the NNLO corrections.

\subsection{Inclusive cross sections and differential distributions for $W^{+} W^{-}$production}

The $W^{+} W^{-}$cross section has been measured at the LHC by both ATLAS and CMS at centreof-mass energies of $7 \mathrm{TeV}$ [44, 45], $8 \mathrm{TeV}$ [43, 46, 47] and $13 \mathrm{TeV}$ [48, 49], agreeing well with the respective SM predictions at NNLO QCD accuracy. In order to suppress the enormous background from top-quark pairs, typical fiducial cuts imply a jet veto. Consequently, higher-order effects are quite different for inclusive results [27] (see Figure 1) and predictions within fiducial phasespace regions [28]: Whereas the loop-induced gluon-gluon contribution amounts to only about one third of the $\mathscr{O}\left(\alpha_{\mathrm{s}}^{2}\right)$ effects in the inclusive case, it dominates if a jet veto is applied, and the genuine corrections to the $q \bar{q}$ channel become even negative. In Figure 4 we show distributions in the dilepton invariant mass, the $p_{\mathrm{T}}$ of the dilepton system and the azimuthal angle between the two leptons. By and large the $\mathrm{NLO}^{\prime}+g g$ approximation, which was considered the best prediction before full NNLO results were known, reproduces the NNLO result quite well. However, we find 

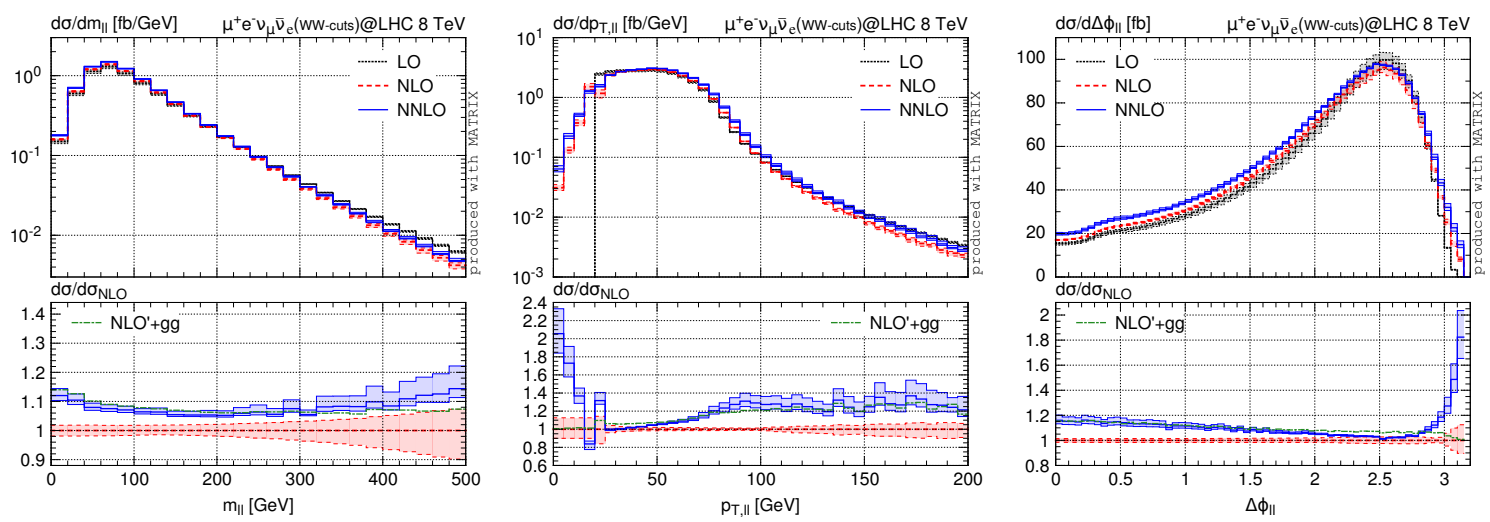

Figure 4: Distributions in the dilepton invariant mass (left), the $p_{\mathrm{T}}$ of the dilepton system (center) and the azimuthal angle between the two leptons (right) are shown. The applied phase-space cuts are inspired by the ATLAS analysis [43], but we do not apply any lepton-isolation criteria with respect to hadronic activity.

shape distortions of up to about $10 \%$ throughout. In phase-space regions that imply the presence of QCD radiation, $\mathrm{NLO}^{\prime}+g g$ cannot approximate the shapes of full NNLO corrections.

\subsection{Inclusive cross sections for $W^{ \pm} Z$ production}

The inclusive $W^{ \pm} Z$ cross section has been measured with good precision at the LHC by ATLAS and CMS at centre-of-mass energies of $7 \mathrm{TeV}[50,51]$ and $8 \mathrm{TeV}[52,51]$. Also early measurements at $13 \mathrm{TeV}[53,54]$ by ATLAS and CMS are already available. The agreement with theory predictions is significantly improved by including the recently calculated NNLO corrections [29] (see Figure 1), in particular for LHC Run 1 data. As for $W^{ \pm} \gamma$ production, the large corrections are explained by a radiation zero, here in the leading helicity amplitudes at Born level, which is overcome only beyond LO.

\section{Summary}

In this contribution, we have presented the current status of the MATRIX code, which allows for the computation of NNLO QCD corrections to a number of colour-singlet production processes with one or two Higgs and vector bosons in the final state. The most relevant physics results obtained with this code for vector-boson pair production processes have been summarized by presenting both total cross sections and distributions in the fiducial phase spaces. By and large, the NNLO corrections significantly improve the agreement with the experimental measurements.

MATRIX is being prepared for publication in the near future. Besides the inclusion of resummation at small transverse momenta through NNLL for all the available colour-singlet processes, which is currently under validation, other developments are possible. These involve the inclusion of electroweak corrections, higher-order corrections to the gluon-induced channels, and the extension of the code to deal with coloured final states.

\section{Acknowledgments}

This research was supported in part by the Swiss National Science Foundation (SNF) under contracts 200020-141360, 200021-156585, CRSII2-141847, BSCGI0-157722 and PP00P2- 
153027, and by the Kavli Institute for Theoretical Physics through the National Science Foundation's Grant No. NSF PHY11-25915.

\section{References}

[1] D. A. Kosower, Phys. Rev. D57 (1998) 5410-5416, [hep-ph/9710213].

[2] A. Gehrmann-De Ridder, T. Gehrmann, and E. W. N. Glover, JHEP 09 (2005) 056, [hep-ph/0505111].

[3] A. Daleo, T. Gehrmann, and D. Maitre, JHEP 04 (2007) 016, [hep-ph / 0612257$].$

[4] J. Currie, E. W. N. Glover, and S. Wells, JHEP 04 (2013) 066, [arXiv : 1301 . 4693].

[5] G. Somogyi, Z. Trocsanyi, and V. Del Duca, JHEP 06 (2005) 024, [hep-ph/ 0502226 ].

[6] V. Del Duca, C. Duhr, G. Somogyi, F. Tramontano, and Z. Trocsanyi, JHEP 04 (2015) 036, [arXiv:1501.07226].

[7] V. Del Duca, C. Duhr, A. Kardos, G. Somogyi, and Z. Trocsanyi, arXiv:1603.08927.

[8] M. Czakon, Phys. Lett. B693 (2010) 259-268, [arXiv: 1005 . 0274].

[9] M. Czakon, Nucl. Phys. B849 (2011) 250-295, [arXiv: 1101.0642 ].

[10] M. Czakon and D. Heymes, Nucl. Phys. B890 (2014) 152-227, [arXiv: 1408 . 2500 ].

[11] S. Catani and M. Grazzini, Phys.Rev.Lett. 98 (2007) 222002, [hep-ph/ 0703012 ].

[12] R. Bonciani, S. Catani, M. Grazzini, H. Sargsyan, and A. Torre, Eur. Phys. J. C75 (2015), no. 12 581, [arXiv:1508.03585].

[13] R. Boughezal, C. Focke, X. Liu, and F. Petriello, Phys. Rev. Lett. 115 (2015), no. 6062002 , [arXiv:1504.02131].

[14] J. Gaunt, M. Stahlhofen, F. J. Tackmann, and J. R. Walsh, JHEP 09 (2015) 058, [arXiv:1505.04794].

[15] G. Bozzi, S. Catani, D. de Florian, and M. Grazzini, Nucl.Phys. B737 (2006) 73-120, [hep-ph/0508068].

[16] D. de Florian and M. Grazzini, Nucl.Phys. B616 (2001) 247-285, [hep-ph / 0108273 ].

[17] S. Catani, L. Cieri, D. de Florian, G. Ferrera, and M. Grazzini, Nucl.Phys. B881 (2014) 414-443, [arXiv:1311.1654].

[18] S. Catani and M. Grazzini, Eur.Phys.J. C72 (2012) 2013, [arXiv: 1106.4652 ].

[19] S. Catani, L. Cieri, D. de Florian, G. Ferrera, and M. Grazzini, Eur.Phys.J. C72 (2012) 2195, [arXiv:1209.0158].

[20] F. Cascioli, P. Maierhöfer, and S. Pozzorini, Phys.Rev.Lett. 108 (2012) 111601, [arXiv:1111.5206].

[21] T. Gehrmann and L. Tancredi, JHEP 02 (2012) 004, [arXiv: 1112 . 1531].

[22] T. Gehrmann, A. von Manteuffel, and L. Tancredi, JHEP 09 (2015) 128, [arXiv: 1503.04812 ].

[23] M. Grazzini, S. Kallweit, D. Rathlev, and A. Torre, Phys.Lett. B731 (2014) 204-207, [arXiv:1309.7000].

[24] M. Grazzini, S. Kallweit, and D. Rathlev, JHEP 07 (2015) 085, [arXiv: 1504 . 01330].

[25] F. Cascioli, T. Gehrmann, M. Grazzini, S. Kallweit, P. Maierhöfer, A. von Manteuffel, S. Pozzorini, D. Rathlev, L. Tancredi, and E. Weihs, Phys.Lett. B735 (2014) 311-313, [arXiv: 1405.2219 ]. 
[26] M. Grazzini, S. Kallweit, and D. Rathlev, Phys. Lett. B750 (2015) 407-410, [arXiv:1507.06257].

[27] T. Gehrmann, M. Grazzini, S. Kallweit, P. Maierhöfer, A. von Manteuffel, S. Pozzorini, D. Rathlev, and L. Tancredi, Phys.Rev.Lett. 113 (2014) 212001, [arXiv: 1408 . 5243].

[28] M. Grazzini, S. Kallweit, S. Pozzorini, D. Rathlev, and M. Wiesemann, JHEP 08 (2016) 140, [arXiv:1605.02716].

[29] M. Grazzini, S. Kallweit, D. Rathlev, and M. Wiesemann, Phys. Lett. B761 (2016) 179-183, [arXiv:1604.08576].

[30] M. Grazzini, S. Kallweit, D. Rathlev, and M. Wiesemann, JHEP 08 (2015) 154, [arXiv:1507.02565].

[31] NNPDF Collaboration, R. D. Ball et al., JHEP 1504 (2015) 040, [arXiv: 1410 . 8849].

[32] ATLAS Collaboration, G. Aad et al., Phys. Rev. D87 (2013), no. 11 112003, [arXiv: 1302 . 1283]. [Erratum: Phys. Rev.D91,no.11,119901(2015)].

[33] CMS Collaboration, S. Chatrchyan et al., Phys. Rev. D89 (2014), no. 9092005 , [arXiv: 1308.6832 ].

[34] CMS Collaboration, S. Chatrchyan et al., JHEP 10 (2013) 164, [arXiv: 1309.1117 ].

[35] ATLAS Collaboration, G. Aad et al., Phys. Rev. D93 (2016), no. 11 112002, [arXiv:1604.05232].

[36] CMS Collaboration, V. Khachatryan et al., JHEP 04 (2015) 164, [arXiv: 1502.05664 ].

[37] CMS Collaboration, V. Khachatryan et al., Phys. Lett. B740 (2015) 250-272, [arXiv:1406.0113].

[38] ATLAS Collaboration, G. Aad et al., JHEP 03 (2013) 128, [arXiv:1211.6096].

[39] CMS Collaboration, S. Chatrchyan et al., JHEP 01 (2013) 063, [arXiv:1211.4890].

[40] ATLAS Collaboration, ATLAS-CONF-2013-020.

[41] ATLAS Collaboration, G. Aad et al., Phys. Rev. Lett. 116 (2016), no. 10 101801, [arXiv:1512.05314].

[42] CMS Collaboration, CMS-PAS-SMP-16-001.

[43] ATLAS Collaboration, G. Aad et al., arXiv:1603.01702.

[44] ATLAS Collaboration, G. Aad et al., Phys.Rev. D87 (2013), no. 11 112001, [arXiv: 1210.2979 ].

[45] CMS Collaboration, S. Chatrchyan et al., Eur. Phys. J. C73 (2013), no. 102610 , [arXiv:1306.1126].

[46] ATLAS Collaboration, M. Aaboud et al., arXiv:1608.03086.

[47] CMS Collaboration, V. Khachatryan et al., Eur. Phys. J. C76 (2016), no. 7 401, [arXiv:1507.03268].

[48] ATLAS Collaboration, ATLAS-CONF-2016-090.

[49] CMS Collaboration, CMS-PAS-SMP-16-006.

[50] ATLAS Collaboration, G. Aad et al., Eur. Phys. J. C72 (2012) 2173, [arXiv: 1208 . 1390].

[51] CMS Collaboration, CMS-PAS-SMP-12-006.

[52] ATLAS Collaboration, G. Aad et al., Phys. Rev. D93 (2016) 092004, [arXiv: 1603.02151 ].

[53] ATLAS Collaboration, M. Aaboud et al., arXiv:1606.04017.

[54] CMS Collaboration, CMS-PAS-SMP-16-002. 